\title{
Atuação dos profissionais de educação física em um centro de atenção psicossocial: Percepção de usuários e trabalhadores
}

\author{
Performance of physical education professionals in a psychosocial care center: Perception of users \\ and worker
}

Desempeño de profesionales de educación física en un centro de atención psicosocial: Percepción de usuarios y trabajadores

Recebido: 19/07/2021 | Revisado: 25/07/2021 | Aceito: 27/07/2021 | Publicado: 02/08/2021

\author{
Taynara Poliana Gonçalves de Melo \\ ORCID: https://orcid.org/0000-0001-6802-0313 \\ Universidade de Pernambuco, Brasil \\ E-mail: taynaragoncalvesdemelo@gmail.com \\ Paolo Porciuncula Lamb \\ ORCID: https://orcid.org/0000-0002-6712-0168 \\ Secretaria de Saúde do Recife, Brasil \\ E-mail:paoloplamb@gmail.com \\ Clarissa Figueredo Braga \\ ORCID: https://orcid.org/0000-0002-3185-6253 \\ Escola de Governo de Saúde Pública de Pernambuco, Brasil \\ E-mail:clarissafb8@hotmail.com \\ Maria Deisyelle Sibaldina da Silva Almeida \\ ORCID: https://orcid.org/0000-0001-8997-0334 \\ Universidade de Pernambuco, Brasil \\ E-mail: mariadeisyelle@gmail.com \\ Mylena Félix dos Santos \\ ORCID: https://orcid.org/0000-0001-9803-3983 \\ Universidade de Pernambuco, Brasil \\ E-mail: mylenafelixsantos@gmail.com \\ Williany Isis Santos \\ ORCID: https://orcid.org/0000-0003-4640-7984 \\ Universidade de Pernambuco, Brasil \\ E-mail:willianyisis@gmail.com \\ Maria Jucineide Lopes Borges \\ ORCID: https://orcid.org/0000-0003-0095-9003 \\ Centro de Atenção Psicossocial. Secretaria de Saúde. Prefeitura do Recife, Brasil \\ E-mail:juciborges@hotmail.com
}

\begin{abstract}
Resumo
A pesquisa teve como objetivo conhecer a atuação do PEF através da percepção de usuários e trabalhadores em um CAPS do município de Recife/PE. Trata-se de uma pesquisa exploratória, estudo de caso com uma abordagem qualitativa. Participaram do estudo: dois PEF, quatro usuários e dois trabalhadores do CAPS. Optou-se por trabalhar com o método de entrevista semiestruturada. Os dados coletados foram verificados por meio da Análise de Conteúdo. Após a análise emergiram quatro tópicos: grupo movimento no CAPS, o corpo e uso de psicofármacos, trabalho interprofissional e saída dos PEF do CAPS. A atuação do PEF no CAPS é vista como um valioso recurso terapêutico para os usuários. É por meio das práticas corporais que se promove maior conhecimento corporal, integração do usuário com o meio e reinserção social. Portanto, a saída dos PEF acarretou uma perda para os dispositivos de saúde mental e quebra do processo terapêutico dos usuários.

Palavras-chave: Saúde mental; Educação física; Relações interprofissionais; Sistema Único de Saúde.

Abstract

The research aimed to know the performance of PEF through the perception of users and workers in a CAPS of Recife / PE. It is an exploratory research, case study with a qualitative approach. Two PEF, four users and two CAPS workers participated in the study. We chose to work with the semi-structured interview method. The collected data were verified through Content Analysis. After the analysis four topics emerged: group movement in CAPS, body and use of psychotropic drugs, interprofessional work and PEF exit from CAPS. PEF's performance in CAPS is seen as a valuable therapeutic resource for users. It is through body practices that greater body knowledge, user integration with the environment and social reintegration is promoted. Therefore, the departure of PEF led to a loss to mental health devices and break the therapeutic process of users.
\end{abstract}


Keywords: Mental health; Physical education; Interprofessional relations; Health Unic System.

Resumen

La investigación tuvo como objetivo conocer el desempeño de PEF a través de la percepción de usuarios y trabajadores en un CAPS de Recife / PE. Es una investigación exploratoria, estudio de caso con un enfoque cualitativo. Dos PEF, cuatro usuarios y dos trabajadores de CAPS participaron en el estudio. Elegimos trabajar con el método de entrevista semiestructurada. Los datos recopilados se verificaron a través del análisis de contenido. Después del análisis surgieron cuatro temas: movimiento grupal en CAPS, cuerpo y uso de drogas psicotrópicas, trabajo interprofesional y salida de PEF de CAPS. El rendimiento de PEF en CAPS se considera un recurso terapéutico valioso para los usuarios. Es a través de las prácticas corporales que se promueve un mayor conocimiento del cuerpo, la integración del usuario con el medio ambiente y la reintegración social. Por lo tanto, la partida de PEF condujo a una pérdida de dispositivos de salud mental y a romper el proceso terapéutico de los usuarios.

Palabras clave: Salud mental; Educación física; Relaciones interprofesionales; Sistema Único de Salud.

\section{Introdução}

A Reforma Psiquiátrica Brasileira surgiu com estreita relação com o Movimento da Reforma Sanitária, e teve como objetivo a quebra da centralidade do procedimento psiquiátrico e biomédico que separavam e demarcavam parte da população considerada doente mental como incapaz de compartilhar sua vida na sociedade. Para superar esse modelo, a Reforma Psiquiátrica empreendeu um conjunto complexo de transformações relacionadas à concepção de saúde e à de doença mental: "movimento que passa a reivindicar transformações das relações entre cultura e loucura, até então demarcadas pela normatização" (Lobosque, 2009).

$\mathrm{O}$ primeiro movimento relacionado à assistência psiquiátrica Brasileira, também conhecido como Movimento dos Trabalhadores em Saúde Mental (MTSM), ganhou força no final dos anos 70, quando profissionais recém-formados encontraram um cenário de descaso e violência nos hospitais psiquiátricos (Amarante, 2018). Foi um movimento muito importante, pois pode ser considerado como um conjunto de práticas de liberdade, na medida em que começou a produzir novas formas de pensar, trabalhar e organizar o campo da saúde (Venturine, 2010).

No ano de 2001, após 12 anos de tramitação no congresso, foi aprovada a Lei 10.216/2001 (Brasil, 2001) conhecida como a "Lei da Reforma Psiquiátrica", que dispõe sobre a proteção e os direitos das pessoas portadoras de transtornos mentais, e redireciona o modelo assistencial em saúde mental.

Com a conquista da Reforma Psiquiátrica surgem os serviços substitutivos chamados de Centros de Atenção Psicossocial (CAPS) (Brasil, 2001), que têm como objetivo o cuidar e o acolher de forma integral as pessoas que apresentam desordem psíquica, possibilitando desenvolver atividades que ultrapassam as tradicionais consultas e prescrições de medicamentos, tirando o foco da doença, e esse cuidado é feito no próprio território dos usuários.

Os profissionais que atuam nos CAPS possuem diversas formações e integram uma equipe multiprofissional. São considerados profissionais de nível superior obrigatórios: enfermeiros, médicos, psicólogos, assistentes sociais, terapeutas ocupacionais, pedagogos ou outros possíveis para as atividades oferecidas nos CAPS (Brasil, 2002). Entre esses possíveis trabalhadores está o profissional de educação física (PEF). Os profissionais inseridos nos CAPS desenvolvem diversas atividades terapêuticas como: oficinas terapêuticas, atividades comunitárias, expressivas e artísticas, atividades domiciliares e familiares (Brasil, 2002) entre outras.

Como forma de superar o modelo tradicional e ampliando o campo de atuação do PEF, em 2012, o Conselho Federal de Educação Física (CONFEF) lançou a Resolução nº 230/2012, que dispõe sobre a especialidade do PEF na área de saúde mental. O artigo $3^{\circ}$ deixa explícito que:

A Especialidade em Educação Física na área da Saúde Mental, incluindo a Atenção Psicossocial, destina-se ao atendimento dos transtornos mentais e do comportamento, incluindo aqueles decorrentes do uso e dependência de substâncias psicoativas, tendo como princípios o Sistema Único de Saúde - SUS, a Reforma Psiquiátrica, em sua perspectiva desinstitucionalizante, e as diretrizes da Política Nacional de Saúde Mental (CONFEF, 2012). 
Diversos estudos apontam sobre os benefícios da atividade física no cuidado aos usuários que estão inseridos em dispositivos de saúde mental. Para Takeda (2006), a atividade física deve ser utilizada como ferramenta para estimular a socialização dos usuários nos CAPS, e contribuir na promoção e na manutenção da saúde. De acordo com Wachs (2009), a atividade física pode vir a ser utilizada como instrumento de mediação no tratamento da saúde mental por fazer parte do cuidado multiprofissional. Este é considerado importante no processo, pois apresenta papel potencializador na promoção da saúde, tendo em vista que os usuários necessitam serem vistos e cuidados na sua integralidade, sem a dissociação corpo e mente.

No ano de 2002, os PEF foram inseridos em alguns dispositivos de saúde mental, além desses profissionais desenvolverem suas atividades no Programa Academia da Cidade (PAC), passaram a distribuir-se também entre os CAPS (Soares et al., 2016).

O município do Recife dispõe de 17 CAPS, que estão divididos em: três CAPSi, para crianças em sofrimento psíquico; seis CAPSad, para indivíduos devido uso abusivo de álcool e outras drogas; e oito CAPS, para adultos com transtorno mental (Secretaria de Saúde, 2019).

No momento, os dispositivos de saúde mental encontravam-se sem a atuação do PEF, devido a uma decisão da classe por questões políticas. Dessa forma, enquanto PEF e Residente em saúde mental, a escolha do objeto de estudo se deu a partir da inquietação em relação à saída desses profissionais dos CAPS, uma vez que a atuação dessa categoria se mostra muito necessária para esses dispositivos de saúde. Portanto, a pesquisa teve como objetivo conhecer a percepção de usuários e trabalhadores sobre a atuação dos PEF em um Centro de Atenção Psicossocial do município de Recife/PE.

\section{Metodologia}

Este estudo caracteriza-se como pesquisa exploratória e estudo de caso, utilizando abordagem qualitativa. A abordagem qualitativa é adequada aos estudos da história, das representações e crenças, das relações, das percepções e opiniões, ou seja, dos produtos das interpretações que os humanos fazem durante suas vidas (Minayo, 2011).

O estudo incluiu a participação de oito sujeitos: dois PEF, quatro usuários e dois trabalhadores do CAPS Esperança do município do Recife/PE, este é um serviço que atende usuários com transtornos mentais graves e persistentes.

Como instrumento de coleta de dados utilizou-se o método de entrevista semiestruturada. Gil (2008) destaca que esse tipo de entrevista permite que o entrevistador retome a questão original ao perceber desvios, ao passo que o entrevistado tem a liberdade de falar abertamente sobre o assunto elencado. $O$ procedimento da coleta de dados foi realizado por meio de um roteiro, elaborado de forma distinta para os PEF, usuários e trabalhadores do CAPS. Os dados foram registrados através de gravador de voz e, posteriormente, transcritos na íntegra.

Os dados foram analisados pela técnica de análise do conteúdo que, segundo Bardin (2011), define-se como um conjunto de técnicas de análise de comunicação que são empregadas para obter, com procedimentos sistemáticos e objetivos de descrição do conteúdo das mensagens, indicadores (quantitativos ou não) que permitam a inferência de conhecimentos relativos às condições de produção/recepção dessas mensagens. Foram realizados os seguintes procedimentos: A) Leitura de materiais da comunidade científica; B) Identificação e comparação das ideias e dos sentidos de cada resposta; C) Definição de núcleos de sentido (significados atribuídos pelos sujeitos); D) Criação de hipótese.

Após a leitura exaustiva das gravações transcritas, surgiram quatro categorias de análise: o grupo movimento no CAPS, o corpo e uso de psicofármacos, o trabalho interprofissional e a saída dos PEF dos CAPS.

A pesquisa foi aprovada pelo Comitê de Ética em Pesquisa da Universidade de Pernambuco em 15 de Outubro de 2019, CAAE 19355919.2.0000.5207. Os participantes foram informados quanto aos objetivos, procedimentos e privacidade das 
informações, e assinaram o Termo de Consentimento Livre e Esclarecido (TCLE). No decorrer dos resultados e discussão os participantes serão identificados pelas nomenclaturas PEF M, PEF F, usuário P, usuário R, trabalhador J e trabalhador E.

\section{Resultados e Discussão}

\section{Grupo movimento no CAPS}

Nos CAPS do Município de Recife foram inseridos PEF do Programa Academia da Cidade (PAC), que é um programa de promoção da saúde com foco nas atividades físicas, lazer e alimentação saudável. O programa foi implementado em 2002, a fim de proporcionar saúde e bem-estar aos munícipes em espaços públicos (Recife, 2009). Entre as atividades desenvolvidas pelo PEF no CAPS, destaca-se o grupo movimento, considerado como um recurso terapêutico no atendimento para pessoas com transtorno mental e/ou necessidades decorrentes do uso de crack, álcool e outras drogas. De acordo com Cruz (2013), o objetivo do grupo movimento é desenvolver atividades físicas e ser um recurso complementar ao projeto terapêutico dos usuários dos CAPS.

Os PEF que participaram da entrevista descreveram quais atividades realizavam no grupo movimento: "Atividades lúdicas, desenvolvia atividades com bola, com arco, jogos... fazia muitas atividades com músicas, aí usava dança, mas de forma mais lenta... aí fazia aula de relaxamento, fazia aula com eles de pilates" (PEF M); "Ginástica localizada, trabalhava aeróbica com eles, trabalhava um pouco de dança, trabalhava aerobox, [...] antes trabalhava esportes, mas o esporte era mais falado, videos" (PEF L).

A maioria dos participantes da pesquisa consideram importante a prática corporal no CAPS, realizado através do grupo movimento, o mesmo foi identificado como um grupo diferenciado em comparação aos demais ofertados pelas diversas categorias profissionais, conforme relatos a seguir: "Eu acho o grupo movimento os PEF, o empenho deles e a paciência que ele tem com o grupo portador de transtorno mental muito bom" (Usuário R). "Era sempre grupos para movimentar [...] tirar aquela inércia, imobilidade...aquela coisa parada” (Usuário P).

O grupo movimento tinha parte lúdica de estar se divertindo, ouvindo uma música, estar se movendo no ritmo daquela música, é... um grupo diferente, é o diferencial [...] É uma oportunidade de trabalhar a coordenação, o movimento, a noção mesmo do corpo, contato com o corpo. Existe estímulo para uma atividade física, para um hábito saudável (Trabalhador E).

Nota-se a valorização das atividades desenvolvidas pelos PEF no grupo movimento no CAPS e, de acordo com Roble (2012), o trabalho do PEF expõe diferentes e novas formas de fazer o cuidado em saúde mental, colocando o usuário como agente participante e ativo em seu tratamento, resgatando no interior do sujeito as relações que o mesmo possui com seu corpo, utilizando essa relação como forma de tratamento e de reinserção na sociedade. Durante a entrevista surge o posicionamento de um usuário:

"Atualmente eu estou indo ao parque da Jaqueira, ai faço exercício lá [...] aí tem esse aplicativo agora para você agendar, que mostra o nome, o RG, aí você tem 40 minutos para fazer exercício, tem em vários lugares" (Usuário P), corroborando com a publicação de Roble (2012).

Para além das atividades realizadas no CAPS, e seguindo na perspectiva da reforma psiquiátrica, os entrevistados relataram que os PEF buscavam incentivar os usuários a acessar e realizar práticas corporais no PAC, como finalidade de promover a reinserção social e produção de sua autonomia de cuidado. De acordo com Paranhos-Passos e Aires (2013), a reinserção e a reabilitação seriam os dois principais conceitos na área da saúde mental: “A gente queria mostrar que atividades em outro espaço era prazeroso e eles poderiam fazer isso além do horário do CAPS, com mais frequência, entrar realmente no PAC, no caso" (PEF M).

No entanto, por mais que busque trabalhar na perspectiva de realizar atividades no território, é importante também que o CAPS busque aproximação com os dispositivos que estão inseridos na própria comunidade dos usuários. De acordo com 
Wachs (2009), se o CAPS adota, entre seus princípios, promover um cuidado voltado para fora para a reinserção social, é conveniente buscar aproximação com práticas sociais desenvolvidas nas comunidades nas quais estão inseridos os usuários.

É importante refletir sobre o lugar do PEF e as atividades que o mesmo desenvolve nos CAPS, bem como introduzir os usuários do serviço na construção das atividades propostas, pois não foi evidenciado, durante a análise das entrevistas, sobre essa inserção. Para Ceccim e Bilibio (2007), a educação física pode recolocar a dimensão corpórea da existência subjetiva para a prática cuidadora, "o lugar do desejo e da energia vital que se impulsione ao contato com as sensações [...] um corpo de afetos e de expansão da experiência humana".

\section{O corpo e uso de psicofármacos}

O cenário das práticas de cuidado ofertadas aos usuários dos serviços de saúde mental ainda estão predominantemente associadas ao uso de psicofármacos, principalmente medicamentalizantes, os quais são considerados como únicos em que, ao sujeito, "diagnosticadamente adoecido", não restam muitas escolhas além de adaptar-se ao processo de cuidado ofertado (Bezerra et al. 2016). Contudo, os psicofármacos apresentam agravantes relacionados ao uso prolongado, como sedação, fadiga, perda de memória, sonolência, dificuldade motora, diminuição da atenção, da concentração e dos reflexos (Neto, 2017).

Com base nas entrevistas realizadas, foi possível identificar que o uso prolongado dos psicofármacos reflete numa diminuição da percepção corporal, fazendo com que, muitas vezes, houvesse uma redução no interesse por movimentar-se e manter-se fisicamente ativo. Para Silva (2009), os benzodiazepínicos apresentam efeitos colaterais: podem induzir o sono, reduzir o tônus muscular e coordenação motora; antidepressivos ocasionam alterações da condução cardíaca e hipotensão postural; e estabilizadores de humor influenciam o ganho de peso, a sedação e a ataxia: "Devido as medicações que se toma você fica com um... muitas vezes travado sem poder o corpo funcionar, e devido a essas medicações deixa você o seu corpo ali, se acorda com muito sono" (Usuário P); "Os usuários com transtorno mental, eles se esquecem um pouco do corpo, eles tomam medicações" (Trabalhador J).

Não se pode negar que o uso de psicofármacos faz parte do processo de tratamento dos usuários que possuem sofrimento psíquico. Na sociedade contemporânea, os psicofármacos têm assumindo papel de destaque e, muitas vezes, é considerado como principal meio de cuidado. O homem pós-moderno se vê em meio a um discurso que procura homogeneizá-lo e tratar de seus sintomas de modo universal, sem buscar possíveis significados (Canabarro, 2009).

De acordo com Neto (2017), existem possibilidades de intervenção nesse grupo de indivíduos que faz uso prolongado de psicofármacos e apresentam efeitos colaterais. Uma das possibilidades, por exemplo, seria a administração de medicamentos associada à prática regular de atividades físicas. Em seu estudo foi identificado que a realização de atividade física têm contribuído para amenizar os efeitos colaterais provenientes do uso prolongado desses fármacos.

Os discursos dos entrevistados vão de acordo com o que preconiza os achados na literatura a respeito dos efeitos positivos que as práticas corporais exercem sobre o grupo no CAPS que faz uso de psicofármacos: "A gente lida com um grupo que toma medicação, muitas vezes tem um corpo mais rígido e esse trabalho do PEF contribui para trabalhar essa rigidez, para trabalhar esses sintomas da medicação" (Trabalhadora J); "É uma coisa que faz bem para o seu próprio corpo né, então é uma coisa muito necessário pra quem faz tratamento no CAPS e para as pessoas que tomam medicação, a questão do exercício fisico" (Usuário P).

Fica evidente que o cuidado em saúde mental vai além do tratamento farmacológico. Ele deve agregar outras áreas que possam contribuir com as propostas terapêuticas nos dispositivos de saúde mental. As práticas corporais desenvolvidas pelos PEF apresentaram importantes contribuições para os usuários dos serviços de saúde mental. 


\section{Trabalho Interprofissional}

Os PEF que participaram da entrevista relataram que na maioria das vezes desenvolviam atividades no CAPS acompanhados por terapeuta ocupacional, psicólogo, assistente social, enfermeiro, entre outros, mostrando dessa forma, uma superação do modelo hegemônico e atuando de maneira integrada, havendo reciprocidade e enriquecimento mútuo com outros profissionais.

Os profissionais com diferentes formações na saúde, articulam seu saber com os demais na organização do trabalho, o que possibilita tanto compartilhar as ações, como delegar atividades a outros profissionais, nos moldes de uma prática colaborativa (Peduzzi, 2013). O trabalho em saúde deve ser realizado na perspectiva de aprimorar o diálogo com os demais trabalhadores, discutir os casos que chegam nos serviços e tomar decisões de forma compartilhada: "O PEF, ele faz atividades não só limitadas ao exercício em si, mas a questão de trabalhar em equipe, de fazer atividades juntas, de trocar ideias, uns com os outros" (Trabalhador J); “Acho muito importante essa atuação da gente lá dentro, junto com outros profissionais. Então, como é uma equipe multiprofissional, a gente sempre fazia atividades em conjunto” (PEF M).

Mesmo diante dos efeitos positivos que a atuação interprofissional proporciona para o trabalho na saúde, ainda existe dificuldade de compreensão sobre esse assunto por parte de alguns integrantes da equipe. Foi possível identificar no discurso de um entrevistado que houve discordância para o PEF desenvolver o trabalho em conjunto com uma trabalhadora de enfermagem no CAPS, evidenciando uma fragmentação no cuidado e conhecimento da saúde, necessitando, dessa forma, de mais capacitações acerca da temática. Ainda, o PEF relatou que houve momentos que não conseguiu sair com os usuários do CAPS para alguma praça ou parque no próprio território, pois teria que ir sozinho, sem outro trabalhador do serviço dando suporte. Nessa perspectiva é possível inferir que ainda há dificuldade de coesão entre profissionais de diferentes categorias na realização de trabalho cooperativo, ações coletivas voltadas a tarefas comuns: "No início eu dividia com a enfermeira... No início a gerência da época deixava. Aí, depois cortou” (PEF L). Eu penso que a união de dois técnicos, de duas linhas diferentes traz experiências diferentes de um determinado assunto, que fosse trabalhado no momento do grupo (PEF L).

A gente já foi para Jaqueira (Parque) e foi até andando. A questão de ir andando é questão de segurança deles [...] Questão de medo de atravessar a rua, porque a gente está com eles e cada um tem um problema diferente: questão de medicação, alguns são mais lentificados, outros agitados. Realmente, para uma pessoa só não dava (PEF L).

Peduzzi (2013) demonstra que a prática interprofissional tende a aumentar a resolubilidade dos serviços e a qualidade da atenção à saúde, pois possibilita evitar omissões ou duplicações de cuidados, evitar esperas e adiamentos desnecessários, ampliar e melhorar a comunicação entre os profissionais, bem como o reconhecimento das contribuições específicas de cada área e de suas fronteiras sobrepostas, com a flexibilização dos papéis profissionais. É no cotidiano que os trabalhadores dos dispositivos de saúde mental são convidados a irem ao encontro do novo, a se deixarem afetar pelos saberes construídos, a sempre querer ir além das amarras da formação inicial e buscar integrar-se com os demais trabalhadores, a construir novas possibilidades de atuação.

\section{Saída dos PEF do CAPS}

Em 2002, os PEF foram inseridos no CAPS cumprindo uma carga horária semanal de 30h, e além desses profissionais desenvolverem suas atividades no PAC, passaram a se distribuir também entre o CAPS (Soares et al. 2016) ou com grupos articulados por unidades de saúde em comunidades. Foi a solução encontrada para atender mais pessoas, já que os pólos do PAC não cobrem toda a cidade (Radis, 2011). 
Soares et al., (2016) demonstrou que um dos aspectos negativos é a carga horária de trabalho limitada do PEF no CAPS. O mesmo trabalhava dez horas semanais, supervisionando o grupo movimento, realizando atividades interdisciplinares, participando de reuniões técnicas, além de outras atividades.

Uma pesquisa realizada por Leonidio (2013), cujo objetivo foi analisar a percepção dos PEF sobre o processo de trabalho nos CAPS de Recife, encontrou como desfecho que os PEF acreditam que o seu trabalho poderia ser ainda mais reconhecido, se tivesse a mesma carga horária que os demais técnicos do CAPS e participassem da rotina dos serviços.

No ano de 2017, os PEF do município de Recife passaram a não atuar nos dispositivos de saúde mental. Durante a entrevista realizada com dois PEF, foram relatados os motivos da saída do CAPS, dentre eles: busca de reconhecimento profissional e melhores condições salariais. A tomada de decisão para saída dessa categoria profissional do CAPS foi apoiada pela Associação dos PEF de Pernambuco (APEF-PE): “Trabalhava aqui uma determinada carga horária e a gente queria receber umas coisas, alguns benefícios que os profissionais do CAPS recebiam, proporcional à carga horária que a gente trabalhava. Isso fez com que a gente saísse” (PEF L); “Quando a gente estava dentro do CAPS, a gente não era visto como profissional de saúde. Então, nossa Associação resolveu priorizar o profissional e ele teria que se enquadrar nas categorias dos profissionais que trabalham em CAPS” (PEF M).

A gente não podia evoluir os prontuários com nossa Classificação Brasileira de Ocupação (CBO), que era uma coisa vista por outros profissionais como uma coisa diferente e pra gente também não estava podendo evoluir, só podia por outro profissional. Eu fazia grupo com outro profissional, porque eu não poderia evoluir, que não tinha validade (PEF M).

Corroborando, a pesquisa de Soares (2016), uma PEF que participou da entrevista expressa sua angústia em relação a carga horária no CAPS: “A carga horária da gente era de catorze horas [...] eu mesma estendia várias vezes porque a demanda era muito grande, não dava pra fazer muita coisa em tão pouco tempo, contanto com reuniões, com grupos, e tudo assim” (PEF M).

Os profissionais dos CAPS desenvolviam diversas atividades terapêuticas como: grupo de família, acolhimento, assembleias com usuários, grupos terapêuticos diversos, discussão de casos, apoio matricial, visitas domiciliares, construção de projeto terapêutico singular (PTS) e técnico de referência (TR), este último considerado fundamental para estreitamento do vínculo entre profissional e usuário. Furtado e Miranda (2006) discorrem que o TR constitui um dispositivo de aproximação entre o profissional ou entre a equipe a um determinado número de usuários do serviço, a qual reverbera no PTS.

Em razão da carga horária limitada e dividida com outro programa, os PEF que atuavam nos CAPS tinham suas atividades também limitadas, como pode ser percebido na fala de um trabalhador entrevistado: “Eles não podiam ser técnico de referência, não podia fazer acolhimento, não fazia as outras atividades que um profissional de nível universitário consegue fazer" (Trabalhador E).

O Registro das Ações Ambulatoriais de Saúde (RAAS) foi instituído pela Portaria Federal no 276 em 30 de Março de 2012 pelo Ministério da Saúde, e tem o objetivo de monitorar as ações e serviços de saúde nas Redes de Atenção à Saúde. Os registros que contemplam o RAAS de cada serviço de saúde devem ser enviados para o Sistema de Informação Ambulatorial, preenchidos com o código de cada trabalhador (CBO). Entretanto, diferente de outros trabalhadores, o PEF não possui um CBO próprio para registro nos serviços de saúde mental. Para registrar suas intervenções nos prontuários dos usuários, os PEF usam o CBO de outros trabalhadores e com atividades que não se referiam à educação física (Machado, 2015). Provavelmente, o não reconhecimento profissional foi um dos fatores para a saída do PEF do CAPS.

A percepção dos demais indivíduos que participaram da entrevista, em relação à saída do PEF do CAPS, demonstrou uma perda para o serviço: "Veja, eu acho que para o CAPS foi ruim. Nessa brincadeira a gente já vai com quase dois anos que 
a gente não tem esse profissional" (Trabalhador J); "Eu acho que essa retirada dos PEF do CAPS não foi uma coisa muito legal, porque desde que os PEF saíram ficou faltando um grupo que tivesse é... movimento" (Usuário P).

Como observado na fala dos entrevistados, a saída dos PEF do CAPS foi considerada negativa para os usuários e trabalhadores do referido serviço. Uma fragilidade da Portaria GM n³36/2002 é de considerar como equipe técnica mínima o profissional médico, enfermeiro, assistente social, terapeuta ocupacional e pedagogo, deixando assim, à critério da gestão local, outro profissional necessário ao projeto terapêutico. Talvez isso explica o motivo de ainda estarmos sem esse profissional nos dispositivos de saúde mental do Município do Recife.

Uma crítica importante referente ao processo de saída dos PEF do CAPS é que não houve uma finalização adequada de um vínculo institucional e terapêutico deste profissional com o serviço: "Os usuários que saíram prejudicados, por causa das atividades fisicas que eles faziam, das conversas e brincadeiras que a gente tinha" (PEF L); A gente não teve tempo de conversar, foi uma coisa que a gente teve uma reunião [...] a gente explicou porque estava saindo para os profissionais" (PEF M).

Essa saída brusca de lá... A gente nem teve tempo de explicar, de dizer o porquê de estar saindo. Então, a gente saiu de uma forma que pra eles... Eles não entendem até hoje, porque a gente saiu tão rápido de lá, sem avisar basicamente nada" (PEF M).

De modo geral, pode-se afirmar que a saída do PEF do CAPS do Município do Recife/PE foi uma perda para o serviço e, principalmente, para os usuários. A inserção dos PEF nos dispositivos de saúde mental é uma das alternativas de cuidado que deve ser trabalhada na proposta da reforma psiquiátrica, para que os usuários desses serviços sejam vistos e tratados em sua integralidade (Miranda \& Freire \& Oliveira, 2011). Grigolo (2015) expressa que as relações entre usuários e os profissionais envolvem apego, afetos e vínculos. Portanto, foi possível identificar que essa perda desencadeou uma quebra no processo terapêutico dos usuários.

\section{Considerações Finais}

A atuação do PEF no CAPS é importante e vista como um valioso recurso terapêutico para os usuários do serviço. O grupo movimento no CAPS, coordenado pelo PEF, destaca-se por desempenhar importantes funções no âmbito da saúde mental, dentre elas: promover momentos de interação, diálogos e trocas, realização de atividades físicas, atividades lúdicas e estímulo para uma vida mais ativa fisicamente. É por meio das práticas de atividades físicas que se promove maior conhecimento corporal, integração do usuário com o meio, além de sua reinserção social. Logo, espera-se que o profissional de educação física tenha suas reivindicações por melhores condições de trabalho e reconhecimento profissional atendidas, e que seja inserido de forma integral nos dispositivos de saúde mental. Sugere-se mais estudos nessa área para potencializar a compreensão sobre a importância da atuação do profissional de educação física nesses espaços de saúde.

\section{Referências}

Amarante, P. \& Nunes, M. D. O. (2018). A reforma psiquiátrica no SUS e a luta por uma sociedade sem manicômios. Ciênc. saúde colet, 23 (6).

Bardin, L. (2011). Análise de Conteúdo. Edição 70. São Paulo: LDA.

Bezerra, I. C et al. (2016). Uso de psicofármacos na atenção psicossocial: uma análise à luz da gestão do cuidado. Saúde em debate, 40, 148 -161.

BRASIL. Ministério da Saúde. (2002). Portaria 336/GM, de 19 de Fevereiro de 2002. Ministério da Saúde. Define e estabelece diretrizes para o funcionamento dos Centros de Atenção Psicossocial. Recuperado em 19 de Abril (2019). http://bvsms.saude.gov.br/bvs/saudelegis/gm/2002/prt0336_19_02_2002.html

BRASIL. Ministério da Saúde. (2012). Resolução n 466, de 12 de dezembro de 2012. Aprova normas regulamentadoras de pesquisas envolvendo seres humanos. Brasília: Diário Oficial da União. Recuperado em 13 de Junho (2019) 022https://bvsms.saude.gov.br/bvs/saudelegis/cns/2013/res0466_12_12_2012.html 
Research, Society and Development, v. 10, n. 9, e590109184437, 2021

(CC BY 4.0) | ISSN 2525-3409 | DOI: http://dx.doi.org/10.33448/rsd-v10i9.18437

BRASIL. Ministério da Saúde. (2012). Portaria N 276, de 30 de Março de 2012. Ministério da Saúde. Institui o sistema de Registro das Ações Ambulatoriais de Saúde (RAAS). Recuperado em 24 de Dezembro (2019) http://bvsms.saude.gov.br/bvs/saudelegis/sas/2012/prt0276_30_03_2012.html

Canabarro, R. D. C. S. \& Alves, M. B. (2009). Uma pílula para (não) viver. Revista Mal-Estar e Subjetividade, 9(3), 839-866.

Ceccim, R. B. \& Bilibio, L. F. (2007). Singularidades da Educação Física na saúde: desafios à educação de seus profissionais e ao matriciamento interprofissional. Educação Física e Saúde Coletiva: políticas de formação e perspectivas de intervenção. Porto Alegre: UFRGS, 47-62.

CONFEF. (2012). Resolução CONFEF nº 230, de 16 de Abril de 2012. Dispõe sobre a especialidade Profissional de Educação Física na área da Saúde Mental. Recuperado em 18 de Junho (2019).

https://www.confef.org.br/confef/resolucoes/res-pdf/302.pdf

Furtado, J. P. \& Miranda, L. (2006). O dispositivo "técnicos de referência” nos equipamentos substitutivos em saúde mental e o uso da psicanálise winnicottiana. Revista Latinoamericana de Psicopatologia Fundamental, 9 (3), 508-524.

Gil, A. C. (2008). Métodos e técnicas de pesquisa social. 6. ed. Editora Atlas SA.

Grigolo, T. M. \& Peres, G. M. \& Junior, C. A. G. \& Rodrigues, J. (2015). O projeto terapêutico singular na clínica da atenção psicossocial. Cadernos Brasileiros de Saúde Mental/Brazilian Journal of Mental Health, 7(15), 53-73.

Leonidio, A. C. R et al. (2013). O profissional de Educação Física no Centro de Atenção Psicossocial: percepção dos limites e potencialidades no processo de trabalho. Revista Pesquisas e Práticas Psicossociais, 8(2), 157-165.

Lobosque, A.M. (2011). Debatendo alguns desafios da Reforma Psiquiátrica Brasileira. Ciência \& Saúde Coletiva, 16 (12),4590-4602.

Machado, G. J. (2015). A atuação do professor de educação física nos Centros de Atenção Psicossocial Álcool e Drogas. Tese de Mestrado. Universidade Federal do Espírito Santo.

Miranda, E. D. \& Freire, L. A. \& Oliveira, A. R. C. (2011). Os desafios da Educação Física no Centro de Atenção Psicossocial de Coari (AM) (The challenges of Physical Education in the Psychosocial Care Center at Coari (AM)). Saúde \& Transformação Social/Health \& Social Change, 2(1), 163-169.

Neto, J. A. \& Leite, L. H. I. \& Rocha, P. G. L. (2017). Uso de psicofármacos e práticas corporais para a saúde em um grupo terapêutico. SANARE-Revista de Políticas Públicas, 16(2).

Paranhos-Passos, F. \& Aires, S. (2013). Reinserção social de portadores de sofrimento psíquico: o olhar de usuários de um Centro de Atenção Psicossocial. Physis: Revista de Saúde Coletiva, 23, 13-31.

Peduzzi, M. et al. (2013). Educação interprofissional: formação de profissionais de saúde para o trabalho em equipe com foco nos usuários. Revista da Escola de Enfermagem da USP, 47(4), 977-983.

Radis. Academias da saúde pública. n. 109, p. 12-17. set. 2011.

Roble, O. J. \& Moreira, M. I. B. \& Scagliusi, F. B. (2012). A educação física na saúde mental: construindo uma formação na perspectiva interdisciplinar. Interface-Comunicação, Saúde, Educação, 16, 567-578.

Secretaria Municipal de Saúde. (2019). Centro de Atenção Psicossocial. Dados Recife. Recife.

Secretaria Municipal de Saúde. (2009). Programa Academia da Cidade: Nota técnica. Recife.

Silva, D. M. C. (2009). Avaliação do consumo de medicamentos psicotrópicos no município de Pacatuba. Monografia. Escola de Saúde Pública do Ceará Fortaleza.

Soares, S. \& Brainer, M. \& Silva, C. Leonidio, A. R. \& Lemos, E. (2016). Contribuições e desafios do profissional de Educação Física no Centro de Atenção Psicossocial. Revista Brasileira de Atividade Física \& Saúde, 21(5), 420-430.

Minayo, M. C. S. \& Deslandes, S. F. \& Gomes, R. (2011). Pesquisa social: teoria, método e criatividade: Editora Vozes Limitada.

Takeda, O. H. \& Stefanelli, M. C. (2006). Atividade física, saúde mental e reabilitação psicossocial. Revista Mineira de Enfermagem, 10(2), $171-175$.

Venturini, E. (2010). “O caminho dos cantos": morar e intersetorialidade na saúde mental. Fractal: Revista de Psicologia, 22(3), 471-480.

Wachs, F. \& Fraga, A. B. (2009). Educação física em centros de atenção psicossocial. Revista Brasileira de Ciências do Esporte, 31(1). 